\title{
Perfect No Bake Cookies
}

\author{
Emily Turner
}

\author{
1 stick Butter \\ 2 Cups Sugar \\ 1/2 Cup Milk \\ 1/3 Cup Cocoa Powder \\ 1 Cup Peanut Butter \\ 3 Cups Rolled Oats \\ 1 Tlb Vanilla
}

This No Bake Cookie recipe was not born out of my kitchen, but it has become my own after years and years of making and crafting and perfecting these cookies. I remember one day my dad brought home a recipe for my sisters and I. We were young, not so great in the kitchen, but we loved to bake. As young bakers, we tried this treat, melding together butter, sugar, cocoa powder, peanut butter, rolled oats, and more. Our entire family quickly became obsessed with these little cookies, and although not every batch was perfect, we continued to make our beloved No Bake Cookies, and now I have the recipe practically memorized. Make these cookies for holidays, special occasions, or for not-so-special occasions. Share with ones you love. Expect

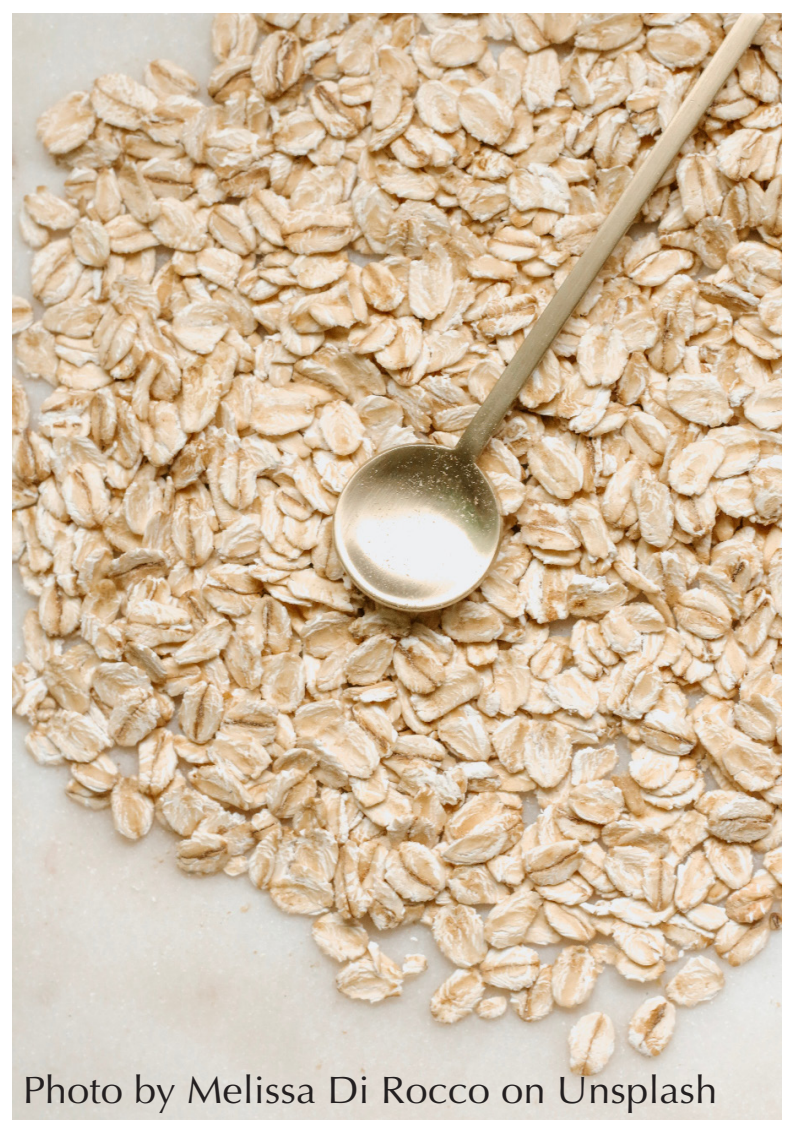
no leftovers. 
The ingredients listed above yields one batch of cookies (number depends on how big you make them), but my sisters and I found that you will need to make a double batch (simply double the ingredients and use a slightly bigger saucepan). Trust me, you'll need to make a double batch.

In a medium saucepan, melt your butter and then add your sugar. You must melt the butter first or else the sugar might burn. Mix until combined and add the milk, cocoa powder, and then stir.

The next step is the most important of this recipe: tread carefully and follow my exact words, and you'll have the perfect batch of No Bake Cookies. Bring the mixture to a boil. It should be more than simmering, but it also shouldn't be boiling over the pan. Stir occasionally. Once the mixture is boiling, stop stirring and let it boil for exactly 60 seconds. Not a second more. Not a second less. If you boil it for longer than 60 seconds, then the cookies will come out dry and crumbly. If you boil it for less than 60 seconds, then the cookies will be runny, and they won't fully set. So make sure to have a timer on hand and set it before the chocolate starts to fully boil!

As soon as the 60 seconds is up, remove the saucepan from the heat. Start by adding the peanut butter. Stir it in and watch as the gooey peanut butter melts into the chocolate, creating ripples of gold throughout the pan (my favorite step). Once combined, stir in the rolled oats and vanilla. To complete the cookies, dollop the dough onto sheets lined with aluminum foil and let harden. (Though I highly recommend trying the batter while it's still warm).

You have successfully made your very own batch of No Bake Cookies! Once hardened, these cookies will be dry to the touch on the outside yet moist and chewy on the inside. These cookies are bite-sized pieces of peanut-butter chocolate heaven. Be sure to share these cookies with your friends and family, but always make sure you save some for yourself. You deserve it. 